\title{
TATE COHOMOLOGY OF PERIODIC $K$-THEORY WITH REALITY IS TRIVIAL
}

\author{
LISBETH FAJSTRUP
}

\begin{abstract}
We calculate the $R O(\mathbb{Z} / 2)$-graded spectrum for Atiyah's periodic $K$-theory with reality and the Tate cohomology associated to it. The latter is shown to be trivial.
\end{abstract}

\section{INTRODUCTION}

Let $K R$ be the periodic $\mathbb{Z} / 2$-spectrum representing Atiyah's Real $K$-theory or $K$-theory with reality [A].

In this note we prove that the generalized Tate spectrum associated to it (in the sense of Greenlees and May [GM]) is trivial. This is quite surprising, since the Tate spectrum for ordinary equivariant $K$-theory is not contractible for any group $G$.

The proof is quite simple however; it just relies on the fact that the generator $\eta$ of $K R\left(\mathbb{R} P^{1}\right)$ is nilpotent. We give a more general statement to emphasize this in 3.1 .

It is a pleasure to thank Marcel Bökstedt and John Greenlees, who shortcut my own lengthy calculation proving Theorem 5.2 by pointing out that the essential ingredient was the fact that $\eta^{3}=0$; and the referee who gave many valuable suggestions to make the exposition more readable.

\section{2. $G$-SPECTRA AND $G$-COHOMOLOGY THEORIES}

In this paper we will be working in the equivariant stable category of [LMS], and we begin by recalling some definitions. We let $G$ be a finite group, since we do not need the Lie group setup of [LMS].

A complete universe $U$ is an infinite dimensional real inner product space with $G$ acting through isometries such that $U$ contains a countably infinite direct sum of regular representations of $G$ as a subspace.

A $G$-spectrum $k_{G}$ indexed on a given $U$ associates a based $G$-space $k_{G}(V)$ to each finite dimensional $G$-subspace $V \subset U$ such that for any two $G$ subspaces $V$ and $W$ of $U$ with $V \subset W$ the usual transitive system of structure maps $k_{G}(V) \rightarrow \Sigma^{V-W} k_{G}(W)$ are $G$-homeomorphisms.

Received by the editors October 12, 1993 and, in revised form, March 24, 1994; originally communicated to the Proceedings of the AMS by Thomas Goodwillie.

1991 Mathematics Subject Classification. Primary 55N15, 55N20, 55R91; Secondary 55P91, 55P42, 55R25.

$K e y$ words and phrases. $K$-theory with reality, Tate cohomology, $G$-spectrum. 
Let $U=\bigoplus\left(V_{i}\right)^{\infty}$ for a set of distinct irreducible representations $V_{i}$. Then $R O(G, U)$ is the free abelian group generated by the $V_{i}$.

Given a $G$-spectrum $k_{G}$ indexed on $U$, we define the associated $R O(G, U)$ graded homology and cohomology theory:

For any virtual representation $a=V-W$ with $V$ and $W$ in $R O(G, U)$ there are sphere $G$-spectra $S^{a}=\sum^{-W} S^{V}$, and we let

$$
k_{G}^{a}(X)=\left[X \wedge S^{-a}, k_{G}\right]^{G} \text { and } k_{a}^{G}(X)=\left[S^{a}, X \wedge k_{G}\right]^{G}
$$

for any $G$-spectrum $X$. For a $G$-space $Y$, let $k_{G}^{a}(Y)=k_{G}^{a}\left(\sum^{\infty} Y\right)$ and similarly for homology; here $\sum^{\infty} Y$ is the $R O(G, U)$-graded suspension spectrum of $Y$ as in [LMS, p. 14]. These theories have suspension isomorphisms $k_{G}^{V}(X) \cong k_{G}^{V \oplus W}\left(S^{W} \wedge X\right)$ and similarly for homology, induced by the structure maps in the spectrum.

On the other hand, given an $R O(G, U)$-graded cohomology theory on $G$ spectra indexed over $U$ (for a definition of this, see [May, Chapter 19], or [LMS, p. 34]), there is a spectrum which classifies this theory as above. Furthermore if the cohomology theory is only defined on $G$-spaces, it has an extension to $G$-spectra, which is unique up to nonunique isomorphism, and hence it gives rise to a classifying $G$-spectrum.

\section{The TATE SPECTRUM FOR SOME $\mathbb{Z} / 2$-SPECTRA}

In [GM] a generalized Tate cohomology theory for a $G$-spectrum $k_{G}$ is introduced for any compact Lie group $G$. We repeat this construction for the convenience of the reader. Let $X_{+}$be the disjoint union of the $G$-space $X$ with a fixed base point and let $E G$ be a contractible free $G$-space. Let $\widetilde{E G}$ be the unreduced suspension of $E G$. Then there is a cofibering

$$
E G_{+} \rightarrow S^{0} \rightarrow \widetilde{E G} \text {. }
$$

Let $F\left(E G_{+}, k_{G}\right)$ be the function $G$-spectrum of maps from $E G_{+}$to $k_{G}$. The projection $E G_{+} \rightarrow S^{0}$ induces a map of $G$-spectra

$$
\varepsilon: k_{G}=F\left(S^{0}, k_{G}\right) \rightarrow F\left(E G_{+}, k_{G}\right) \text {. }
$$

Smashing $\varepsilon$ with the cofibering (1) yields the following map of cofiberings of $G$-spectra:

$$
\begin{array}{cccc}
k_{G} \wedge E G_{+} & k_{G} & \longrightarrow & k_{G} \wedge \widetilde{E G} \\
\varepsilon \wedge 1 \downarrow & { }^{\prime} \downarrow & \varepsilon \wedge 1 \\
F\left(E G_{+}, k_{G}\right) \wedge E G_{+} & \longrightarrow F\left(E G_{+}, k_{G}\right) \longrightarrow F\left(E G_{+}, k_{G}\right) \wedge \widetilde{E G}
\end{array}
$$

The Tate $G$-spectrum associated to $k_{G}$ is then the spectrum in the lower right hand corner of this diagram: $t\left(k_{G}\right)=F\left(E G_{+}, k_{G}\right) \wedge \widehat{E G}$. The associated homology and cohomology theories are the Tate homology and cohomology of $k_{G}$.

When $G=\mathbb{Z} / 2$ and $k_{G}$ is a ring spectrum, the following special case of [GM, §16] gives a method for calculating the upper right-hand corner of (2).

Let $m$ denote the trivial real $m$-dimensional representation of $\mathbb{Z} / 2$ and let $k \xi$ be $\mathbb{R}^{k}$ with involution given by multiplication by -1 . We allow $m$ and $k$ to be infinite and let $\mathbb{R}^{\infty}$ have the direct limit topology as usual. 
When $G=\mathbb{Z} / 2$, our universe will always be $U=\infty \xi \oplus \infty$.

A filtration of $\widetilde{E \mathbb{Z} / 2}$ can be obtained from the fact that $B(\infty \xi) / S(\infty \xi)$ is a model for $\widetilde{E \mathbb{Z} / 2}$, which implies that the one point compactifications $S^{p \xi}$ give a filtration. We get canonical isomorphisms in homology for any $X$ and for any subspace $V$ of our universe; in cohomology this works for finite $X$ :

$$
\begin{gathered}
\left(k_{\mathbb{Z} / 2} \wedge \widetilde{E \mathbb{Z} / 2}\right)^{V}(X) \cong \operatorname{colim}\left(k_{\mathbb{Z} / 2} \wedge S^{p \xi}\right)^{V}(X) \cong \operatorname{colim}\left(k_{\mathbb{Z} / 2}^{V \oplus \xi}(X)\right), \\
\left(k_{\mathbb{Z} / 2} \wedge \widetilde{E \mathbb{Z} / 2}\right)_{V}(X) \cong \operatorname{colim}\left(k_{\mathbb{Z} / 2} \wedge S^{p \xi}\right)_{V}(X) \cong \operatorname{colim}\left(k_{V-p \xi}^{\mathbb{Z} / 2}(X)\right) .
\end{gathered}
$$

Here the maps are induced by the inclusion $S^{p \xi} \rightarrow S^{(p+1) \xi}$. Studying these maps a little closer, we find that the maps giving the latter colimits may also be described as multiplication by a certain element of $k_{\mathbb{Z} / 2}^{\xi}\left(S^{0}\right)$, namely the image of the identity element of $k_{\mathbb{Z} / 2}^{0}\left(S^{0}\right)$ under the isomorphism $k_{\mathbb{Z} / 2}^{0}\left(S^{0}\right) \rightarrow k_{\mathbb{Z} / 2}^{\xi}\left(S^{\xi}\right)$ composed with the map induced by the inclusion of $S^{0}$ in $S^{\xi}$

$$
i^{*}: k_{\mathbb{Z} / 2}^{\xi}\left(S^{\xi}\right) \rightarrow k_{\mathbb{Z} / 2}^{\xi}\left(S^{0}\right) \text {. }
$$

This element is called the Euler class of $\xi$ and is denoted $\chi_{\xi}$. This leads to the following lemma:

Lemma 3.1. Let $k_{\mathbb{Z} / 2}^{*}(-)$ be an $R O(\mathbb{Z} / 2, U)$-graded cohomology theory such that $\chi_{\xi}$ is nilpotent and the classifying spectrum $k_{\mathbb{Z} / 2}$ is a ring spectrum. Then the projection $p$ of $E \mathbb{Z} / 2_{+}$to $S^{0}$ induces a weak $\mathbb{Z} / 2$ homotopy equivalence $p^{*}: k_{\mathbb{Z} / 2} \rightarrow F\left(E \mathbb{Z} / 2_{+}, k_{\mathbb{Z} / 2}\right)$, and the Tate spectrum $t\left(k_{\mathbb{Z} / 2}\right)$ is trivial.

Proof. The long exact sequence induced by the cofibration (1) and the fact that $k_{\mathbb{Z} / 2}^{0}\left(E \mathbb{Z} / 2_{+} \wedge X\right)=\left[X, F\left(E \mathbb{Z} / 2_{+}, k_{\mathbb{Z} / 2}\right)\right]^{\mathrm{Z} / 2}$ implies that for a proof of the first statement it suffices to see that $k_{\mathbb{Z} / 2}^{*}(\widetilde{E \mathbb{Z} / 2} \wedge X)=0$ for $X$ compact. There is a Milnor $\lim ^{1}$-exact sequence [M]

$$
0 \rightarrow \lim ^{1} k_{\mathbb{Z} / 2}^{*-1}\left(S^{p \xi} \wedge X\right) \rightarrow k_{\mathbb{Z} / 2}^{*}(\widetilde{E \mathbb{Z} / 2} \wedge X) \rightarrow \lim k_{\mathbb{Z} / 2}^{*}\left(S^{p \xi} \wedge X\right) \rightarrow 0 .
$$

The maps in the inverse system are the same as in (3), namely

$$
i^{*}: k_{G}^{*}\left(S^{k \xi} \wedge X\right) \rightarrow k_{G}^{*}\left(S^{(k-1) \xi} \wedge X\right)
$$

or equivalently $i^{*}: k_{G}^{*-k \xi}(X) \rightarrow k_{G}^{*-k \xi+\xi}(X)$, and they are multiplication by $\chi_{\xi}$. Since $\chi_{\xi}$ is nilpotent by assumption, we conclude that the inverse limit and the $\lim ^{1}$ are both trivial.

For the second part of the lemma, observe that we have just seen that the middle arrow in (2) is a $\mathbb{Z} / 2$-homotopy equivalence; the leftmost arrow is always an equivalence and hence we see that $t\left(k_{\mathbb{Z} / 2}\right)$ is $\mathbb{Z} / 2$-equivalent to $k_{\mathbb{Z} / 2} \wedge \widetilde{E \mathbb{Z} / 2}$.

From (4) it now follows that the Tate homology groups $t\left(k^{\mathbb{Z} / 2}\right)_{V}(X)$ are trivial for any $X$, since this is a direct limit over multiplication with the nilpotent element $\chi_{\xi}$, and hence the spectrum classifying this theory, $t\left(k_{\mathbb{Z} / 2}\right)$, is trivial.

\section{THE REAL $K$-THEORY SPECTRUM}

In the following we state some facts about the $R O(\mathbb{Z} / 2, U)$-graded spectrum representing Atiyah's Real $K$-theory. 
Definition 4.1 (Atiyah [A]). A Real vector bundle is a complex vector bundle $E \downarrow X$, where $(E, \tau)$ and $(X, t)$ are $\mathbb{Z} / 2$ spaces such that the involutions commute with the projection map and with $\tau$ antilinear, i.e. $\tau(z e)=\bar{z} \tau(e), z \in$ $\mathbb{C}$.

The Real $K$-theory of $(X, t), K R(X)$, is then the Grothendieck group of the isometry classes of Real vector bundles over $X$.

The kernel of the complex dimension map gives reduced $K R$-theory, $\widetilde{K R}(X)$, and this is the theory which we study here. The dimension map is split, so $K R(X)$ is isomorphic to $\widetilde{K R}(X) \oplus \mathbb{Z}$.

The classifying space for reduced Real $K$-theory is $B U$ with $\mathbb{Z} / 2$-action given by complex conjugation [tD]. A model for this is the infinite Grassmannian $G R\left(\mathbb{C}^{\infty}\right)=\bigcup G R_{n}\left(\mathbb{C}^{\infty}\right)$ of complex subspaces of $\mathbb{C}^{\infty}$ with action induced by complex conjugation on $\mathbb{C}^{\infty}$.

An $n$-dimensional Real bundle is a $\left(\mathbb{Z} / 2 \times{ }_{\alpha} U(n)\right)$-bundle in the sense of tom Dieck [tD], where $\alpha: \mathbb{Z} / 2 \rightarrow \operatorname{Aut}(U(n))$ is complex conjugation, whereas equivariant $K$ or $K O$ would have trivial $\alpha$ in this description. Another way of stating this is that a Real bundle is a $\mathbb{Z} / 2$-bundle with total group $\Gamma=$ $\mathbb{Z} / 2 \times_{\alpha} U(n)$, structural group $U(n)$ and fiber $\mathbb{C}$ in the sense of [LMS, p. 175].

In [A], Atiyah introduces a $\mathbb{Z} \oplus \mathbb{Z}$-graded cohomology theory on $\mathbb{Z} / 2$-spaces as follows:

$$
K R^{p, q}(X, Y)=K R\left(X \times B^{p, q}, X \times S^{p, q} \cup Y \times B^{p, q}\right)=K R\left(S^{p \xi+q} \wedge X / Y\right)
$$

where $B^{p, q}$ and $S^{p, q}$ are the ball, respectively the sphere of the representation $p \xi+q$, and $K R(X, A)=K R(X / A)$.

This theory has two kinds of periodicity, namely 1-1-periodicity: $K R^{p, q}(X)$ $\cong K R^{p+1, q+1}(X),[\mathrm{A}$, Theorem 2.3], and 8-periodicity:

$$
K R^{p, q}(X) \cong K R^{p+8, q}(X)
$$

[A, Theorem 3.10]. Thus it can be extended to negative $p$ and $q$.

As in $\S 3$, we work in the complete universe $U=\infty \xi \oplus \infty$. For $K R^{*}$ to be an $R O(\mathbb{Z} / 2, U)$-graded cohomology theory, we want suspension isomorphisms $K R^{V}\left(S^{V} \wedge X_{+}\right) \cong K R(X)$ for any subspace $V$ in $U$. For this to be true we define

$$
K R^{V}\left(X_{+}\right)=K R^{-p,-q}(X)
$$

for $V=p \xi \oplus q$. The reader should beware of the change of signs, since this is quite confusing.

We let $K R$ denote the $\mathbb{Z} / 2$-spectrum representing this theory, and we state some of Atiyah's results in this language.

Let $H$ be the canonical line bundle over $\mathbb{C} P^{1}$ and let $b=[H]-1 \in$ $K R\left(\mathbb{C} P^{1}\right) \cong K R^{-(\xi \oplus 1)}(*)$. The $1-1$ periodicity theorem states

Theorem 4.2 (Atiyah). Multiplication by $b$ induces an equivariant homotopy equivalence between $K R \wedge S^{V}$ and $K R \wedge S^{V-\xi \oplus 1}$ for $V \subset U$ and $\xi \oplus 1 \subset V$.

Proof. By the 1-1 periodicity theorem [A, Theorem 2.3], multiplication with $b$ gives an isomorphism between $K R^{V}(X)$ and $K R^{V-\xi \oplus 1}(X)$. Since this is true for any $\mathbb{Z} / 2$-space, we get the desired $\mathbb{Z} / 2$ homotopy equivalence.

Now let $\alpha(\lambda)$ be the generator of $K R^{8 \xi}(*)$. The 8-periodicity theorem is 
Theorem 4.3 (Atiyah). Multiplication by $\alpha(\lambda)$ induces an equivariant homotopy equivalence between $K R$ and $K R \wedge S^{8 \xi}$.

Proof. The Real periodicity theorem [A, Theorem 3.10] says, that multiplication by $\alpha(\lambda)$ induces an isomorphism between $K R(X)$ and $K R^{8 \xi}(X)$ for any $X$.

We state some consequences of the periodicity theorems: For $p \geq q$

$$
K R \wedge S^{p \xi \oplus q} \simeq K R \wedge S^{(p-q) \xi}
$$

and for any $X$

$$
K R^{p \xi \oplus q}(X) \cong K R^{(p-q) \xi}(X)
$$

Now $\sum^{-V}\left(S^{V} \wedge K R\right) \simeq K R$ and $K R \wedge S^{V} \simeq \sum^{-(\xi \oplus 1)}\left(K R \wedge S^{V}\right)$, so

$$
K R \wedge S^{p \xi \oplus q} \simeq \sum^{-(p \xi \oplus p)}\left(S^{p \xi \oplus q} \wedge K R\right) \simeq \sum^{-(p-q)}(K R) .
$$

For $p \leq q$, by the same arguments

$$
K R \wedge S^{p \xi \oplus q} \simeq \sum^{-(q-p) \xi}(K R) \simeq K R \wedge S^{q-p}
$$

and

$$
K R^{p \xi \oplus q}(X) \cong K R^{q-p}(X)
$$

The fixed points of $K R$ yield periodic real $K$-theory, $K O$, since $K R(Y)=$ $K O(Y)$ for any $\mathbb{Z} / 2$-fixed space $Y$ and nonequivariantly $K R$ is homotopy equivalent to periodic $K$-theory. Thus Real $K$-theory provides an example of a non-split $G$-spectrum, since a splitting [GM, Definition 0.2] is a homotopy equivalence $K U \rightarrow K O \rightarrow K U$ mapping the naive $G$-spectrum $K R$ considered nonequivariantly $(K U)$ through the fixed point spectrum $(K O)$ and via the inclusion to $K U$. This can never be a homotopy equivalence, one reason being that $\pi_{6}(K O)$ is trivial and $\pi_{6}(K U)$ is not.

\section{Calculation of $t(K R)$}

We will see that $K R$ fulfills the conditions of 3.1 .

Let $\eta$ be the generator of $K R^{\xi}\left(S^{0}\right) \cong K R\left(\mathbb{R} P^{1}\right) \cong K O\left(\mathbb{R} P^{1}\right)$, which represents the reduced Hopf bundle over $\mathbb{R} P^{1}$.

Lemma 5.1. Let $i: S^{0} \rightarrow S^{p \xi}$ be the inclusion. Then the induced map $i^{*}$ : $K R^{*}\left(S^{p \xi}\right) \rightarrow K R^{*}\left(S^{0}\right)$ is multiplication by $(-\eta)^{p}$.

Proof. In [A, 3.2], Atiyah studied a map induced by the inclusion of the ball $\left(B(p \xi)_{+},+\right)$in $\left(B(p \xi)_{+}, S(p \xi)_{+}\right)$. The induced map in $K R$-theory is equivalent to the map induced by $i: S^{0} \rightarrow S^{p \xi}$, via equivariant homotopy equivalences of the spaces, and Atiyah proved that it is multiplication by $(-\eta)^{p}$.

This is all we need to prove our main theorem.

Theorem 5.2. The Tate spectrum $t(K R)$ is trivial, and the projection of $E \mathbb{Z} / 2_{+}$ to $S^{0}$ induces a $\mathbb{Z} / 2$-homotopy equivalence between $K R$ and $F\left(E \mathbb{Z} / 2_{+}, K R\right)$. Proof. This will follow from 3.1 once we show that $\chi_{\xi}$ is nilpotent, since $K R$ is a ring spectrum via tensor product of real bundles. Now Lemma 5.1 implies that $\chi_{\xi}=-\eta$ and $\eta^{3}=0$ by [A].

By the discussion in [GM, $\S 5]$, this implies that the homotopy orbit spectrum for $K R$ is equivalent to the homotopy fixed point spectrum, which by $(5.2)$ is the fixed point spectrum $K O$, but we will not go into that here. 


\section{REFERENCES}

[A] M. F. Atiyah, K-theory and reality, Quart. J. Math. Oxford Ser. (2) 17 (1966), 367-386.

[tD] T. tom Dieck, Faserbündel mit Gruppenoperation, Arch. Math. (Basel) 20 (1969), 136-143.

[GM] J. P. C. Greenlees and J. P. May, Generalized Tate, Borel and coBorel cohomology, Preprint, Univ. of Chicago, 1993.

[LMS] L. G. Lewis, J. P. May and M. Steinberger, Equivariant stable homotopy theory, Lecture Notes in Math., vol. 1213, Springer, 1986.

[May] J. P. May, Lecture notes from the NSF/CBMS regional research conference on equivariant homotopy and cohomology theory, in Fairbanks Alaska, August, 1993.

[M] J. W. Milnor, Axiomatic homology theory, Pacific J. Math. 12 (1962), 337-341.

Department of Mathematics and Computer Science, Aalborg University, Fr. BajersVEJ 7E, 9220 Aalborg Øst, Denmark

E-mail address: fajstrup@iesd.auc.dk 